\title{
Highly expressed SLC35F2 in non-small cell lung cancer is associated with pathological staging
}

\author{
LIANG BU, GUANCHAO JIANG, FAN YANG, JUN LIU and JUN WANG \\ Department of Thoracic Surgery, Peking University People's Hospital, Xicheng, Beijing 100044, P.R. China
}

Received May 11, 2011; Accepted August 16, 2011

DOI: $10.3892 / \mathrm{mmr} .2011 .572$

\begin{abstract}
Homo sapiens solute carrier family 35 member F2 (SLC35F2) is highly homologous to the lung squamous cell cancer-related gene, LSCC3, which is highly expressed in lung squamous cell tumour tissues. However, the clinical implication of the SLC35F2 gene in tumour development and progression remains unclear. An affinity-purified polyclonal antibody raised against the human SLC35F2 peptide was used in the immunohistochemical analysis of a non-small cell lung cancer (NSCLC) tissue microarray of human NSCLC $(n=129)$. SLC35F2 protein was also analysed with the same antibody using Western blotting. Total RNAs were extracted from tumour tissues $(n=43)$ and from laser-dissected tumour cells $(n=9)$. SLC35F2 gene expression was detected by fluorescent real-time quantitative PCR and compared to the expression in the corresponding adjacent normal lung tissues. It was found that both the SLC35F2 protein (by IHC analysis) and the SLS35F2 gene transcripts (by Q-PCR analysis) were expressed at significantly higher levels in the NSCLC tumour tissues than in the corresponding adjacent normal lung tissues ( $\mathrm{p}<0.001$ and $=0.015$, respectively). There was a significant correlation between the SLC35F2 transcript and pathological staging $(\mathrm{r}=0.219, \mathrm{p}=0.029)$, although the correlation between SLC35F2 protein and the staging was not significant $(r=0.175)$. SLC35F2 was highly expressed in NSCLC tissues and the levels of expression, in particular the levels of the SLC35F2 transcript, were associated with NSCLC pathological staging. SLC35F2 appears to have a significant prognostic value in NSCLC.
\end{abstract}

Correspondence to: Dr Jun Wang, Department of Thoracic Surgery, Peking University People's Hospital, No. 11 Xizhimen South Street, Xicheng, Beijing 100044, P.R. China

E-mail: drdolphin2008@hotmail.com

Abbreviations: SLC35F2, Homo sapiens solute carrier family 35 member F2; NSCLC, non-small cell lung cancer; TMA, tissue microarray; LCM, laser capture microdissection; IHC, immunohistochemical staining

Key words: solute carrier family 35 member F2, non-small cell lung cancer, polyclonal antibody, tissue microarray, laser capture microdissection

\section{Introduction}

Non-small cell lung cancer (NSCLC) is a group of heterogeneous clinical entities sharing molecular and cellular origins which, however, have different clinical behaviours. The most common NSCLCs are squamous cell carcinoma, large-cell carcinoma and adenocarcinoma. NSCLC is usually not very sensitive to chemotherapy and/or radiation, making surgery an important option for early stage and chemotherapy for advanced (metastatic) NSCLC.

The Homo sapiens solute carrier family 35 member F2 (SLC35F2) (Genebank No. NM 017515) was first reported by Stankovic et al (1) in the study of ataxia telangiectasia in 1997. Subsequently, the molecule was also found to be highly expressed in adult salivary glands by Nishimura et al (2). SLC35F2 was reported to be involved in human blood-brain barrier construction by Bangsow et al (3), but its function is otherwise unknown.

The SLC35F2 gene is located on chromosome 11q22.3 which is close to RAB39, a member of the Ras oncogene family. Recently, it has been demonstrated that the RAB39 gene is closely related to the genesis of a variety of malignant tumours, such as lung, oesophageal, bladder, stomach and rectal cancers. However, the clinical implication of the SLC35F2 gene in tumour development and progression remains unclear. SLC35F2 is highly homologous to another lung squamous cell cancer-related gene, LSCC3, which was found to be highly expressed in lung squamous cell tumour tissues compared to normal lung tissues (4). In the present study, we investigated the pattern of expression of SLC35F2, at the protein and message levels, and deduced the possible relationship between SLC35F2 expression and the pathological staging of NSCLC.

\section{Materials and methods}

Preparation, purification and identification of the antiSLC35F2 polyclonal antibody. First, the structure of the SLC35F2 protein was analyzed by TMHMM Server (http:// www.cbs.dtu.dk/services/TMHMM/). We compared the multiple sequences of SLC35F2 and detected antigenicity, hydrophilicity, hydrophobicity and the quality of surface exposure by DNAstar Lasergene 7.1 (DNASTAR Inc., Madison, WI, USA) as described (5). Four segments of SLC35F2 protein amino acid sequences were selected (named peptide 1,2,3 and 4 , respectively). After the specificity was verified in the protein 
Table I. Baseline characteristics of the NSCLC cases.

\begin{tabular}{lcc}
\hline Characteristics & Immunohistochemical staining & Quantitative real-time PCR \\
\hline Total no. & 129 & 52 \\
Male & 96 & 38 \\
Female & 33 & 14 \\
Age (years) & & $24-81$ \\
Range & $24-82$ & $61.6 \pm 10.5$ \\
Mean & $62.5 \pm 11.2$ & 30 \\
Histological types, n & & 22 \\
Squamous cell carcinoma & 69 & \\
Adenocarcinoma & 63 & 5 \\
Degree of differentiation & & 23 \\
Well & 16 & 24 \\
Moderate & 62 & \\
Poor & 51 & 14 \\
TNM staging & & 13 \\
I & 45 & 4 \\
II & 26 & 4 \\
III & 46 & \\
IV & 12 & \\
\hline
\end{tabular}

TNM staging was according to the UICC (Union for International Cancer Control), 2009 edition.

database, these polypeptides were synthesised and coupled with keyhole limpet hemocyanin (KLH) (Xinran Biotech Inc., Shanghai, China). The KLH-conjugated peptides 1, 2, 3 and 4 were mixed (1:1:1:1) before immunising New Zealand rabbits (Peking University Experimental Animal Center). The immunisation was according to an established method (6). Fourteen days after the second immunisation, blood samples were collected from ear marginal veins for titer test. When the titer reached $\geq 1: 10^{5}$, the rabbits were sacrificed and blood serum samples were collected. The polypeptide antibody titer was detected by ELISA assay (7), and it was considered to be positive if ten times higher than pre-immunisation. The antiSLC35F2 polyclonal antibody was purified by immuno-affinity chromatography. Then, the antibody was quantitated and detected by SDS-PAGE electrophoresis to identify the purity. The proteins were collected from human NSCLC tissues for Western blotting (8). The dilution of the SLC35F2 polyclonal antibody was 1:1,000. The paraffin sections of human NSCLC tissues were prepared for immunohistochemical staining (8). Citric acid retrieval solution (Dingguo Biotech, Inc., Shanghai, China) was used for antigen retrieval by microwave, and the dilution of the SLC35F2 polypeptide antibody was 1:300.

Immunohistochemical staining of the NSCLC tissue microarray. The paraffin-embedded specimens were prepared and stained by H\&E. The clinical and pathological information of the patients is listed in Table I. The sites enriched with typical tumour cells were selected to prepare the tissue microarray wax blocks: $2 \mathrm{~mm} /$ tissue lump, 60 tissue lumps/wax block and $4 \mu \mathrm{m} /$ slice. Immunohistochemical staining of SLC35F2 was carried out as described previously (two pathologists from the Department of Pathology of Peking University People's Hospital for the report of immunohistochemical staining). Citric acid antigen retrieval solution was used for antigen retrieval by microwave, 5 min $x 2$ times, and the dilution of SLC35F2 polypeptide antibody was 1:300. The specific staining of SLC35F2 protein in the cytoplasm of alveolar epithelial cells and tumour cells was observed in five high-power fields (x40). The staining was scored as follows:,$-<5 \%$ cells were stained light yellow or exhibited non-specific staining;, $\pm 5-10 \%$ cells were stained light yellow;,$+ 10-25 \%$ cells were stained yellow;,$++ 25-50 \%$ cells were stained yellow or brown;,$+++>50 \%$ cells were stained brown or dark brown (8).

Detection of the SLC35F2 gene transcript by quantitative real-time $P C R$. Fresh tumour and corresponding adjacent normal lung tissues ( $5 \mathrm{~cm}$ away from the tumour) were frozen in liquid nitrogen immediately after removal, and placed into storage at $-80^{\circ} \mathrm{C}$. Total RNA was extracted from 43 suitable samples out of 52 by TRIzol extraction. Total RNA $(1 \mu \mathrm{g})$ was reversed to cDNA using the Promega reverse transcription system. NSCLC cells were obtained from 9 cases of tumour tissues by laser capture microdissection (LCM). The LCM specimens were embedded in oxytetracycline (OTC). Frozen sections $(10-\mu \mathrm{m})$ were tiled onto EVA film glass slides, dried immediately by cold wind and soaked for $10 \mathrm{sec}$ in DEPC water. The slides were then immersed in hematoxylin (dissolved in DEPC water) for $1 \mathrm{~min}$ in order to stain the nucleus. This was followed by extensive washing using alkaline DEPC water ( $\mathrm{pH}$ 8.0). Following drying, the frozen sections were placed on the loading platform of the Leica LMD6000 laser capture micro-dissection system (Leica Co., Germany). The capture procedure was carried out as instructed by the manufacturer. Between 8,000-10,000 tumour cells from the identified fields were isolated from each specimen. RNA was extracted from these cells using similar methods as 
Table II. Polypeptide sequences for the SLC35F2 antibody preparation.

\begin{tabular}{llc}
\hline Name & \multicolumn{1}{c}{ Sequence } & $\begin{array}{c}\text { Amino acid } \\
\text { no. }\end{array}$ \\
\hline peptide 1 & meadspagpgapeplaegaaaefssllrrik & 31 \\
peptide 2 & gadilagrednsgsdvl & 17 \\
peptide 3 & cstptrtaepaessv & 15 \\
peptide 4 & lglkleenlqeth & 13 \\
\hline
\end{tabular}

mentioned in the previous sections. The SLC35F2 gene transcripts in the NSCLC cells and corresponding adjacent normal lung tissues were detected by real-time quantitative PCR with SYBR Green (Applied Biosystems, Inc., USA). GAPDH was used as the internal control. The nucleotide sequences of the primers were as follows: GAPDH, 5'-TGCATCCTGCACCA CCAACT-3' and 5'-AACACGGAAGGCCATGCCAG-3'; SLC35F2, 5'-ACATTTCTCCATCACTCATTCC-3' and 5'-GACTTAGTTTCTTGAGCTGATGC-3'. The product size was $225 \mathrm{bp}$. The reaction system volume was $50 \mu 1$, the concentrations of each component were in accordance with ABI Power SYBR Green PCR Master Mix (Applied Biosystems, Inc.) guidance manual and the annealing temperature was $58^{\circ} \mathrm{C}$ for 28 cycles.

$\triangle \mathrm{CT}$ (normal lung tissue $)=\mathrm{CT}(\mathrm{SLC} 35 \mathrm{~F} 2$ in normal lung tissue) - CT (GAPDH in normal lung tissue)

$\triangle \mathrm{CT}(\mathrm{NSCLC})=\mathrm{CT}(\mathrm{SLC} 35 \mathrm{~F} 2$ in NSCLC $)-\mathrm{CT}(\mathrm{GAPDH}$ in NSCLC).

The gene expression of SLC35F2 in tissues was represented by $2^{-\Delta \mathrm{CT}} . \Delta \Delta \mathrm{CT}=\Delta \mathrm{CT}$ (NSCLC) $-\Delta \mathrm{CT}$ (normal lung tissue). The fold changes in SLC35F2 gene expression in tumour tissues over normal lung tissues were represented by $2^{-\Delta \Delta C T}$.

Statistical analysis. SPSS 11.5 software and the paired t-test were used for statistical analysis. $p<0.05$ was considered statistically significant, and the Wilcoxon test for non-parametric evaluation of two related samples was used. Correlation and factor analyses were run to determine the clinicopathological features. The degree of immunohistochemical staining and SLC35F2 gene expression levels in tumour tissues, and factors with correlations were selected for variance analysis.

\section{Results}

Preparation of the anti-SLC35F2 polyclonal antibody. The sequences selected to synthesise four polypeptides and to prepare the antibody are shown in Table II. Factors such as hydrophobicity, antigenicity and the quality of surface exposure were considered. The four peptides, with a respective purity at $89.7,84.6,88.3$ and $86.1 \%$ resulted in the antibody titer, which reached $1: 10^{5}$ after three immunisations. The polyclonal antibody was purified with resultant preparation of the immunoglobulin at $1.1 \mathrm{mg} / \mathrm{ml}$ as tested by BCA assay. In order to detect the ability to recognise a linear antigenic epitope and the specificity of this polypeptide antibody, proteins from NSCLC (squamous cell carcinoma) tissues were separated by SDS-PAGE, transferred to a nitrocellulose (NC) membrane
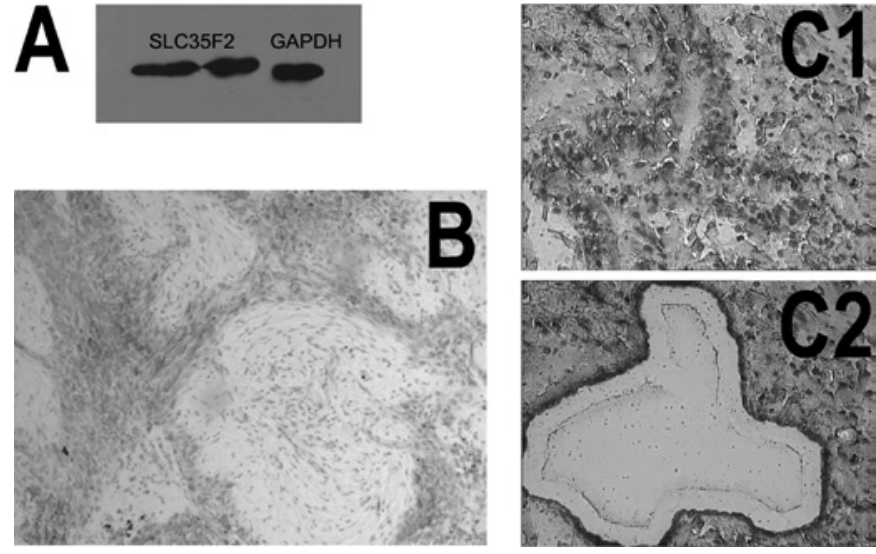

Figure 1. (A) Specificity of the SLC35F2 polypeptide antibody detected by Western blotting. The molecular weight of the SLC35F2 protein is 41,211 $\mathrm{Da}$; molecular weight of the GAPDH protein is 42,030 Da. (B) SLC35F2 was highly expressed in human NSCLC tissues detected by immunohistochemistry. Magnification, x20. (C) NSCLC cells isolated by laser capture microdissection. $\mathrm{C} 1$, before microdissection; $\mathrm{C} 2$, after microdissection.

and detected by the SLC35F2 polypeptide antibody. The antiSLC35F2 polypeptide antibody was able to specifically bind with SLC35F2 in the human NSCLC tissues (Fig. 1A).

SLC35F2 was highly expressed in the human NSCLC tissues. As shown in Fig. 1B, SLC35F2 was highly expressed in human NSCLC tissues compared to normal lung tissues by immunohistochemical staining with the SLC35F2 polypeptide antibody. Analysis using a tissue microarray further revealed that SLC35F2 protein expression in the NSCLC tissues was significantly higher than that in the corresponding adjacent normal lung tissues by paired comparison test $(\mathrm{p}<0.001$, Table III). LCM-purified NSCLC cells from stromal cells showed no change in morphology. Blood vessels, connective tissues, normal alveolar epithelial cells and other non-tumour components successfully remained on the residual slices (Fig. 1C). SLC35F2 transcripts were found to be significantly increased in NSCLC tissue than those in the normal lung tissues (Table IV).

The expression of SLC35F2 was correlated with NSCLC pathological staging. We used both the results from immunohistochemical staining and real-time PCR to correlate the expression of SLC35F2 with the pathological staging of the patients. The correlation analysis of four clinical pathological features (gender, pathological type, pathological differentiation and pathological staging) and SLC35F2 protein expression levels in the NSCLC tissues from the immunohistochemical staining results showed there was a low level of correlation between pathological staging and SLC35F2 expression, with a correlation coefficient of 0.175 . Factor analysis showed that 93.3\% of SLC35F2 expression in NSCLC tissues was due to pathological staging and changes in pathological differentiation. The correlation analysis of four clinicopathological features (gender, pathological type, pathological differentiation and pathological staging) and SLC35F2 gene expression levels in NSCLC tissues from the PCR results also showed there was a low level of correlation between pathological staging and SLC35F2 expression, with a correlation coefficient of 0.219. Further variance analysis showed that SLC35F2 gene 
Table III. Immunohistochemical staining results of the SLC35F2 protein.

\begin{tabular}{|c|c|c|c|c|c|}
\hline & $(-)$ & $( \pm)$ & $(+)$ & $(++)$ & $(+++)$ \\
\hline Normal lung tissues & $31(24.0 \%)$ & $5(3.8 \%)$ & $71(55.1 \%)$ & $22(17.1 \%)$ & $0(0 \%)$ \\
\hline NSCLC tissues & $0(0 \%)$ & $4(3.1 \%)$ & $8(6.2 \%)$ & $58(45.0 \%)$ & $59(45.7 \%)$ \\
\hline
\end{tabular}

Table IV. Expression levels of SLC35F2 by quantitative real-time PCR.

\begin{tabular}{|c|c|c|c|}
\hline Method & Expression level & p-value & NSCLC/normal lung tissues \\
\hline \multicolumn{4}{|l|}{ Regular } \\
\hline NSCLC & $0.47 \pm 0.23$ & 0.049 & $718.4 \pm 450.4$ \\
\hline Normal lung tissues & $0.07 \pm 0.01$ & & \\
\hline \multicolumn{4}{|l|}{ LCM } \\
\hline NSCLC cells & $11.97 \pm 4.47$ & 0.028 & $8,763.7 \pm 3,257.1$ \\
\hline Normal lung tissues & $0.003 \pm 0.001$ & & \\
\hline \multicolumn{4}{|l|}{ Total } \\
\hline NSCLC & $2.46 \pm 0.97$ & 0.015 & $2,110.8 \pm 779.6$ \\
\hline Normal lung tissues & $0.006 \pm 0.001$ & & \\
\hline
\end{tabular}

Table V. Correlation of pathological staging and SLC35F2 expression levels in the NSCLC tissues.

\begin{tabular}{lccc}
\hline Stage & $\begin{array}{c}\text { Cases } \\
\mathrm{n}\end{array}$ & $\begin{array}{c}\text { Expression } \\
\text { level }\end{array}$ & $\mathrm{p}$-value \\
\hline Early (stages I-II) & 27 & $0.30 \pm 0.14$ & 0.029 \\
Advanced (stages III-IV) & 25 & $4.80 \pm 1.93$ & \\
\hline
\end{tabular}

expression levels rose with increased staging. This prompted us to divide the patients into early stage lung cancer (stages I and II) and advanced stage lung cancer (stages III and IV) for comparison. The results showed that the SLC35F2 expression was significantly higher in patients with advanced stage compared to those with early stage disease $(\mathrm{p}=0.029$; Table V).

\section{Discussion}

We prepared and purified the SLC35F2 polypeptide antibody in our study. The designed lengths of the three polypeptides in this study were under 20 amino acids; only one was 31 amino acids long; thus, polypeptides with high purity were obtained and an antibody with high quality was prepared. The SLC35F2 polyclonal antibody raised in our study was found to be of high titer, and could identify the linear epitopes of the SLC35F2 protein accurately and specifically by ELISA detection, Western blotting and immunohistochemistry. The antibody was proved to be specific and suitable for staining the protein in both fresh frozen sections and, most importantly, the paraffin-embedded tissue microarray. Significantly high levels of SLC35F2 staining were identified in tumour tissues in comparison to adjacent normal tissues.
We further investigated SLC35F2 gene expression levels using RNA samples prepared with a traditional method (extracted by grinding cancer tissues) and from laser-dissected purified cancer cells. In this study, the level of the SLC35F2 transcript in the NSCLC samples obtained by a standard method was 0.47 , a weak significant rise compared to that in the normal lung tissues $(\mathrm{p}=0.049)$. However, and strikingly, the expression levels of SLC35F2 in the LCM-purified NSCLC cells reached 11.97, a 20-fold increase over the results obtained by the standard method and highly different from that of the normal tissues $(\mathrm{p}=0.028)$. Several studies have reported that laser capture microdissection combined with fluorescent real-time quantitative PCR is a reliable method to test gene expression in specific tissues (9-12). This appears well reflected in the present study.

At present, the role of SLC35F2 in cancer is unclear. Cancer-related studies on SLC35F2 are somewhat scarce. The present study and that of Shen et al (4) are closely related, but the results are somewhat inconsistent. The reasons for the discrepancy between the two studies may be as follows. i) The number of samples in the previous study from Shen et al was small, so there may be bias of the data; ii) the previous study from Shen et al focused on the nucleic acid level. In the present study, however, a new polyclonal antibody with high titer verifying the specificity was used. Our results included not only the nucleic acid level, but also the protein level, and they were consistent with each other; iii) LCM method was used to obtain tumour cells accurately as well as fluorescent real-time quantitative PCR, thus with an increase in accuracy compared to regular tissue RNA extraction and semi-quantitative RT-PCR technology. It was highly reliable. However, further studies are required.

Nevertheless, a large meta-analysis revealed that platinumbased chemotherapy prolongs the median survival time of patients with advanced NSCLC for only approximately 
6 weeks, compared to the best supportive care (13). Within the last decade, a number of new cytotoxic agents, such as paclitaxel, docetaxel, gemcitabine and vinorelbine, have emerged to offer multiple choices for patients with advanced lung cancer. However, each of these regimens confers only a modest survival benefit compared to cisplatin-based therapies $(14,15)$. To overcome these limitations, new therapeutic strategies that rely on agents designed to target specific tumour-associated molecules are under development (16). Our study revealed that SLC35F2 was highly expressed in NSCLC tissues and was associated with the pathological stage. To some extent, the pathological stage of cancer reflects its biological behaviour. This may provide a new therapeutic target for NSCLC treatment in the future.

In summary, the rapid antibody preparation method by the designing of a synthetic polypeptide provides a method for the functional study of a new gene. Using this method, we successfully obtained an SLC35F2 polyclonal antibody and found that the SLC35F2 protein was expressed differentially in NSCLC and corresponding adjacent normal lung tissues at both the protein and nucleic acid levels. In addition, the expression levels were positively correlated with the pathological stage of NSCLC. Currently, we are planning to expand the number of samples, combine experimental results with follow-up data, identify the mechanisms of SLC35F2 differential expression and investigate the role of this differential expression in the development of NSCLC. The understanding of SLC35F2 gene functions may provide new insight into the molecular and cellular role of SLC35F2 in NSCLC and the validity of the molecule as a new target for NSCLC treatment.

\section{Acknowledgements}

This study was supported by a grant from the National Natural Sciences Foundation of China to Professor Jun Wang (30772486). The authors are extremely thankful to all of the patients who provided their samples for this study.

\section{References}

1. Stankovic T, Byrd PJ and Cooper PR: Construction of a transcription map around the gene for ataxia telangiectasia: identification of at least four novel genes. Genomics 40: 267-276, 1997.
2. Nishimura M, Suzuki S and Satoh T: Tissue-specific mRNA expression profiles of human solute carrier 35 transporters. Drug Metab Pharmacokinet 24: 91-99, 2009.

3. Bangsow T, Baumann E and Bangsow C: The epithelial membrane protein 1 is a novel tight junction protein of the bloodbrain barrier. J Cereb Blood Flow Metab 28: 1249-1260, 2008.

4. Shen C, Zhao H and Wang D: Molecular cloning, identification and analysis of lung squamous cell carcinoma-related genes. Lung Cancer 38: 235-241, 2002.

5. Zang LQ, Qiu PX and Xiao R: [Bioinformatics analysis of YZ-2 protein and polyclonal antibody preparation]. Chinese J Cell Mol Immunol 22: 205-207, 2006 (In Chinese).

6. Liu HY, Yang W and Wu ZH: [Cytochrome P450 3A4 epitope analysis and antibody preparation and identification]. Chinese $\mathrm{J}$ Cell Mol Immunol 23: 79-81, 2007 (In Chinese).

7. Li T, Guo H and Wang Y: [Anti-CMTM4 polyclonal antibody preparation, purification and identification]. Chinese J Cell Mol Immunol 24: 41-44, 2008 (In Chinese).

8. Shi S, Zhang YM and Qiu XY: Anti-chemokine-like factor-1 polypeptide antibody preparation, identification and tissue microarray analysis. Acta Academiae Medicinae Sinicae 26: 496-509, 2004.

9. Sugiyama Y, Sugiyama K and Hirai Y: Microdissection is essential for gene expression profiling of clinically resected cancer tissues. Am J Clin Pathol 117: 109-116, 2002.

10. Jacquet R and Hillyer J: Analysis of connective tissues by laser capture microdissection and reverse transcriptase-polymerase chain reaction. Anal Biochem 337: 22-34, 2005.

11. Hao Q, Xu Y and Li WL: The combined application of laser capture microdissection and linear RNA amplification methods in the study of bladder transitional cell carcinoma related genes. Chinese J Exper Surg 22: 864-867, 2005.

12. Feng JZ, Qin LX and Ren N: Loss of heterozygous study of short arm on chromosome 8 in hepatocellular carcinoma cells by laser capture microdissection. Chinese J Exper Surg 23: 284-287, 2006.

13. Non-small Cell Lung Cancer Collaborative Group: Chemotherapy in non-small cell lung cancer: a meta-analysis using updated data on individual patients from 52 randomised clinical trials. Br Med J 311: 899-909, 1995.

14. Schiller JH, Harrington D, Belani CP, Langer C, Sandler A, Krook J, Zhu J and Johnson DH: Comparison of four chemotherapy regimens for advanced non-small-cell lung cancer. N Engl J Med 346: 92-98, 2002.

15. Kelly K, Crowley J, Bunn PA Jr, Presant CA, Grevstad PK, Moinpour CM, Ramsey SD, Wozniak AJ, Weiss GR, Moore DF, Israel VK, Livingston RB and Gandara DR: Randomized phase III trial of paclitaxel plus carboplatin versus vinorelbine plus cisplatin in the treatment of patients with advanced non-small cell lung cancer: a Southwest Oncology Group trial. J Clin Oncol 19: 3210-3218, 2001.

16. Baselga J: Why the epidermal growth factor receptor? The rationale for cancer therapy. Oncologist 7 (Suppl 4): 2-8, 2002. 